\title{
Role of healthcare professionals in cancer screening
}

\author{
Emily WY Tsang *, Michelle CM Chan, Letty HL Chan, Jacqueline PH Chan, SL Lee, MF Tsui
}

Faculty of Medicine, The Chinese University of Hong Kong, Hong Kong

*Corresponding author: emilytsangwy@link.cuhk.edu.hk

Hong Kong Med J 2020;26:546-8

https://doi.org/10.12809/hkmj209148

Screening promotes early detection of cancer in the asymptomatic population so as to provide timely treatment. Screening has been shown to be effective in detecting cervical cancer and colorectal cancer (CRC); however, the effectiveness of breast cancer and prostate cancer screening remains unclear. ${ }^{1,2}$ In this commentary, we discuss the roles of healthcare professionals in screening of two important cancers in Hong Kong: CRC and breast cancer. ${ }^{3-5}$

Colorectal cancer was the most common cancer among men and the second most common among women in Hong Kong in $2018 .^{6}$ Among new cancer cases, CRC accounted for $17 \%$ of them, with a higher prevalence in the older age-group. In 2014 and $2015,90 \%$ of cases were patients aged $\geq 50$ years. Colorectal cancer was the second leading cause of death in 2018, leading to around 2300 deaths, a rate that was relatively stable between 1981 and $2018 .^{7}$ In Hong Kong, breast cancer is the commonest cancer in women, with an incidence of 109.3 per 1000000 population in 2017. Breast cancer is the third leading cause of death for women, but there was no significant change in the age-standardised death rate between 1981 and $2018 .^{8}$

\section{Effectiveness of cancer screening}

The effectiveness of cancer screening is illustrated by lowering mortality. Meta-analyses have shown that mortality rates were decreased by $59 \%$ by the faecal immunohistochemical test for CRC and by $31 \%$ in mammography for breast cancer. ${ }^{9,10}$ However, low participation in screening limits its effectiveness. The participation rate for CRC screening was only 8.3\% in Hong Kong in the period 2017 to 2018 for people born in 1946 to $1955 .{ }^{11}$ Despite the advantages of cancer screening, there are also some drawbacks, including high rates of false positives and false negatives. Overdiagnosis can lead to unnecessary pain and radiation, resulting in negative psychological consequences for misdiagnosed patients. $^{12}$

\section{Barriers to cancer screening}

Major barriers to cancer screening include a lack of medical knowledge and awareness in the general population. In a study in Hong Kong, $<30 \%$ of respondents recognised that early CRC is asymptomatic, and many had poor knowledge of symptoms and screening methods associated with CRC. ${ }^{13}$ Psychological factors, including embarrassment and fear, also contribute to poor screening uptake, whereas a positive attitude towards screening is key to enhance participation and compliance. ${ }^{14}$

\section{Roles of healthcare professionals}

\section{Advocacy}

Healthcare professionals should encourage asymptomatic individuals as well as people at risk to participate in cancer screening. Frontline healthcare professionals, in particular general practitioners (GPs) and community pharmacists, are in a position to identify eligible individuals, as they act as first point of contact for the general population in the community. Because GPs may understand patients' social histories, personalities and attitudes in addition to their physical health conditions and demographics, they can advise their patients as "trusted advisors". ${ }^{15}$ A thorough face-toface explanation of screening and its procedures by healthcare professionals enhances patients' willingness to opt in for screening. In Hong Kong, government-initiated screening programmes also rely on primary healthcare clinics to provide screening venues for community participation, including the CRC Screening Programme. In Western countries, community pharmacists provide updated information on cancer screening in a simple and concise manner. ${ }^{16}$

\section{Patient education}

Healthcare professionals also have a role in educating patients about risk factors for cancers and distinctive features of screening modalities. Perceived risks and health benefits significantly increase the likelihood of CRC screening participation, thus substantiating the need for interventions to educate individuals. Healthcare professionals are held responsible for offering a thorough explanation of CRC screening and addressing patients' concerns, even when different 
individuals have varying values, perceptions, and health beliefs. Patient education not only improves health literacy, but also modifies patients' attitudes and intentions to partake in cancer screening. Physicians may also act on the variables pertinent to behavioural models, such as perceived behavioural control, intention for screening, and attitudinal attributes to encourage screening uptake. ${ }^{17}$

\section{Offering choice for screening modalities}

Healthcare professionals play a crucial role in maintaining an informed decision-making process, in particular by promoting patient autonomy, through offering choices of different screening modalities. For CRC screening, a local study concluded that patients with regret over their initial screening choice were associated with poorer screening compliance, ${ }^{18}$ while a low compliance also stems from limited knowledge of benefits and risks of the screening modalities available. ${ }^{19}$ Increasing evidence also shows higher patient participation and screening adherence with the offer of choice for different screening modalities, with an odds ratio of 2.54 (95\% confidence interval $=2.30-2.82, \mathrm{P}<0.001) .{ }^{20}$ Current modalities of breast cancer screening include mammography and magnetic resonance imaging, as well as patient self-examination. However, population-based screening is still under much debate in Hong Kong. The importance of healthcare professional recommendation of mammographic screening was highlighted by a study, which found that the majority of respondents in Hong Kong had never heard of mammography and therefore did not undergo regular check-ups. ${ }^{21}$

\section{Involvement in screening process}

To maximise the cancer detection rate, healthcare professionals maintain screening quality by following guidelines and undergoing regularly audits. Some documented problems in CRC screening include positive faecal occult blood test results without follow-up and colonoscopy that is unable to reach the cecum or detect important lesions. ${ }^{22}$ Withdrawal time and adenoma detection rate are important quality indicators for CRC screening. ${ }^{23}$ To mitigate inconsistencies in international breast cancer screening guidelines, local quality assurance standards should be established for the existing mammography service in Hong Kong. Psychological support is essential for promoting screening adherence. Patients less satisfied with past screening are more prone to early dropout, consistent refusal, or delayed screening. ${ }^{24}$ Non-pharmacological alternatives, particularly aromatherapy and selfchosen music, reduce anxiety during invasive screening. Healthcare professionals should explain the possibility of false-positive results to prepare patients psychologically before screening and reinforce the benefits of continuous screening after receiving false-positive results. Nurse-led screening can attain similar quality to that of physician-led procedures, with better emotional support..$^{25,26}$

\section{Compliance and monitoring}

Healthcare professionals play an essential role in improving patients' compliance to screening, particularly through giving advice of regular screening. There are multiple methods by which physicians could monitor patients' situation after the first round of screening, for instance through the alert system in patients' electronic medical records during the follow-up period. ${ }^{27}$ In addition, interactive training seminars on achieving shared decisionmaking could be held to boost physicians' intention to prescribe faecal immunohistochemical test and colonoscopy. ${ }^{28}$ Patients could also be reminded to take part in cancer screening via interactive telephone calls which have been demonstrated to be significant in enhancing CRC screening uptake. ${ }^{29} \mathrm{~A}$ nonadherence model could be established by combining six parameters, including sex, history of psychiatric illness, non-adherence ratio, wait time, number of prior missed endoscopies, and education level, to predict patients' non-compliance. ${ }^{30}$ Healthcare professionals could then make use of such a model to encourage target groups with higher probability of non-adherence to continue cancer screening.

\section{Conclusion}

Healthcare professionals play multifaceted roles in promoting cancer screening as advocates, educators, medical experts, quality controllers, and supporters of patients. Interventional roles discussed in this study are limited to individuals already actively engaged in the healthcare system, thus prompting the need for collaboration with public health policy makers for effective outreach. Cancer screening rates vary widely among different socio-economic conditions, suggesting an inequality in screening utilisation. Further research is required to clarify methods to lower barriers for implementing screening programmes for specific high-risk populations, and to promote extensive screening with greater public acceptance.

\section{Author contributions}

All authors contributed to the concept of the study, acquisition and interpretation of the data, drafting of the manuscript, and critical revision of the manuscript for important intellectual content. All authors had full access to the data, contributed to the study, approved the final version for publication, and take responsibility for its accuracy and integrity.

\section{Conflicts of interest}

All authors have disclosed no conflicts of interest. 


\section{Funding/support}

This commentary received no specific grant from any funding agency in the public, commercial, or not-for-profit sectors.

\section{References}

1. Wang $\mathrm{HH}$, Wang J. Implications of evidence-based understanding of benefits and risks for cancer prevention strategy. Hong Kong Med J 2019;25:346-8.

2. Chiu PK, Ng CF. Primum non nocere (first, to do no harm) in prostate biopsy. Hong Kong Med J 2019;25:344-5.

3. Sitt JC, Lui CY, Sinn LH, Fong JC. Understanding breast cancer screening-past, present, and future. Hong Kong Med J 2018;24:166-74.

4. Lam TH, Chan KH, Chan KK, et al. Recommendations on prevention and screening for breast cancer in Hong Kong. Hong Kong Med J 2018;24:298-306.

5. Lam TH, Wong KH, Chan KK, et al. Recommendations on prevention and screening for colorectal cancer in Hong Kong. Hong Kong Med J 2018;24:521-6.

6. Hong Kong Cancer Registry, Hospital Authority, Hong Kong SAR Government. Top ten cancers. Available from: https://www3.ha.org.hk/cancereg/topten.html. Accessed 24 Nov 2020.

7. Centre for Health Protection, Department of Health, Hong Kong SAR Government. Colorectal cancer. Available from: https://www.chp.gov.hk/en/healthtopics/content/25/51. html. Accessed 20 Oct 2020.

8. Centre for Health Protection, Department of Health, Hong Kong SAR Government. Breast cancer. Available from: https://www.chp.gov.hk/en/healthtopics/content/25/53. html. Accessed 14 Oct 2020.

9. Zhang J, Cheng Z, Ma Y, et al. Effectiveness of screening modalities in colorectal cancer: a network meta-analysis. Clin Colorectal Cancer 2017;16:252-63.

10. Nelson HD, Fu R, Cantor A, Pappas M, Daeges M, Humphrey L. Effectiveness of breast cancer screening: systematic review and meta-analysis to update the 2009 U.S. preventive services task force recommendation. Ann Intern Med 2016;164:244-55.

11. Centre for Health Protection, Department of Health, Hong Kong SAR Government. Colorectal cancer screening pilot programme interim report of the screening outcome for participants enrolled between 28 September 2016 and 27 March 2018. Available from: https://www.colonscreen. gov.hk/sites/default/files/pdf/CRCSPP_ProgressReport_ asofMar2018_final.pdf. Accessed 24 Nov 2020.

12. Independent UK Panel on Breast Cancer Screening. The benefits and harms of breast cancer screening: an independent review. Lancet 2012;380:1778-86.

13. Wong GC, Lee KY, Lam KF, Fan SY. Community-based survey of knowledge of, attitudes to and practice of colorectal cancer screening in Hong Kong. J Dig Dis 2017;18:582-90.

14. Cossu G, Saba L, Minerba L, Mascalchi M. Colorectal cancer screening: the role of psychological, social and background factors in decision-making process. Clin Pract Epidemiol Ment Health 2018;14:63-9.

15. Brown LJ, Roeger SL, Reed RL. Patient perspectives on colorectal cancer screening and the role of general practice. BMC Fam Pract 2019;20:109.

16. Prentice A, Marshall S, Vance M, Choglay S, Von Wagner C, Kerrison R. Colorectal cancer screening and the role of community pharmacy. Pharm J 2019;302:7921.

17. Huang J, Wang J, Pang TW, et al. Does theory of planned behaviour play a role in predicting uptake of colorectal cancer screening? A cross-sectional study in Hong Kong. BMJ Open 2020;10:e037619.

18. Wong MC, Ching JY, Chan VC, et al. Regret on choice of colorectal cancer screening modality was associated with poorer screening compliance: a 4-year prospective cohort study. PLoS One 2015;10:e0125782.

19. Young P, Womeldorph C. Colonoscopy for colorectal cancer screening. J Cancer 2013;4:217-26.

20. Wong MC, Ching JY, Chan VC, et al. Informed choice vs. no choice in colorectal cancer screening tests: a prospective cohort study in real-life screening practice. Am J Gastroenterol 2014;109:1072-9.

21. Hong Kong Breast Cancer Foundation. BCR Bulletin. Available from: https://www.hkbcf.org/en/our_research/ main/424/upload/category/59/self/56c19a26d824d.pdf. Accessed 14 Oct 2020.

22. Holden DJ, Jonas DE, Porterfield DS, Reuland D, Harris R. Systematic review: enhancing the use and quality of colorectal cancer screening. Ann Intern Med 2010;152:66876.

23. Gómez V, Wallace BM. Quality indicators for colonoscopy. In: Shaukat A, Allen JI, editors. Colorectal Cancer Screening: Quality and Benchmarks. New York: Springer New York; 2015: 113-21.

24. Duncan A, Turnbull D, Wilson C, et al. Behavioural and demographic predictors of adherence to three consecutive faecal occult blood test screening opportunities: a population study. BMC Public Health 2014;14:238.

25. Jazrawi A, Berglöf A, Axelhed E, Eriksson J, Bergqvist L, Eriksson $S$. Breast examination by mammography nursesis it reliable? Eur J Surg Oncol 2020;46:e51.

26. Joseph J, Vaughan R, Strand H. Effectiveness of nurseperformed endoscopy in colorectal cancer screening: a systematic review. Gastrointest Nurs 2015;13:26-33.

27. Guiriguet C, Muñoz-Ortiz L, Burón A, et al. Alerts in electronic medical records to promote a colorectal cancer screening programme: a cluster randomised controlled trial in primary care. Br J Gen Pract 2016;66:e483-90.

28. Selby K, Cornuz J, Gachoud D, et al. Training primary care physicians to offer their patients faecal occult blood testing and colonoscopy for colorectal cancer screening on an equal basis: a pilot intervention with before-after and parallel group surveys. BMJ Open 2016;6:e011086.

29. Wong MC, Ching JY, Lam TY, et al. Association of interactive reminders and automated messages with persistent adherence to colorectal cancer screening. a randomized clinical trial. JAMA Oncol 2017;3:1281-3.

30. Blumenthal DM, Singal G, Mangla SS, Macklin EA, Chung DC. Predicting non-adherence with outpatient colonoscopy using a novel electronic tool that measures prior non-adherence. J Gen Intern Med 2015;30:72431. 